\title{
Mycolic acid-containing bacteria activate heterologous secondary metabolite expression in Streptomyces lividans
}

\author{
Hiroyasu Onaka ${ }^{1,2}$, Taro Ozaki ${ }^{1}$, Yukiko Mori ${ }^{2}$, Masumi Izawa ${ }^{2}$, Shohei Hayashi ${ }^{1}$ and Shumpei Asamizu ${ }^{1}$ \\ The Journal of Antibiotics (2015) 68, 594-597; doi:10.1038/ja.2015.31; published online 1 April 2015
}

Streptomyces contains 30-40 secondary metabolite biosynthetic gene clusters; however, researchers can normally detect only a fraction of these secondary metabolites from a single strain under pure culture conditions. ${ }^{1}$ This suggests that the majority of these gene clusters are silent or are expressed below detectable levels. The development of heterologous expression techniques has enabled us to express such genes in alternative hosts, but the production level of each compound in heterologous hosts is often low and does not yield a sufficient level of production. To improve the productivity of heterologously expressed secondary metabolites, new methods that activate the silent or poorly expressed secondary metabolite genes are required.

In nature, diverse groups of microorganisms form complex microbial communities. Hence, it is generally believed that co-culture is a more efficient method for secondary metabolite production than a pure culture because two different microorganisms in the same culture will better mimic the natural environment in which microorganisms continuously interact with each other. ${ }^{2}$

We previously reported that bacteria containing mycolic acids in their outer membrane could induce secondary metabolism in a broad range of Streptomyces strains. ${ }^{3}$ Mycolic acids are fatty acids that are important components in forming the highly impermeable outer barrier in Corynebacterineae. ${ }^{4}$ Streptomyces lividans possesses biosynthetic gene clusters for two red pigments, actinorhodin and undecylprodigiosin, in its genome, but they are not produced in pure culture under normal laboratory conditions. However, S. lividans starts to produce these pigments abundantly in co-culture with mycolic acidcontaining bacteria such as Tsukamurella pulmonis. This indicates that the production of these red pigments is induced by the mycolic acidcontaining bacteria. Interestingly, physical contact between the two strains is required for induction. When cultures of S. lividans and T. pulmonis were separated by a membrane that can transfer small molecules but prevents any cell-to-cell interactions, S. lividans did not produce the red pigments, indicating that the induction of gene expression is not mediated by small molecules but rather by physical contact of the two types of bacteria. ${ }^{3}$
Co-culture with T. pulmonis affected secondary metabolite production in over $80.0 \%$ of soil-isolated Streptomyces strains. Co-culture with two other mycolic acid-containing bacteria, Rhodococcus erythropolis and Corynebacterium glutamicum, also changed the secondary metabolite profiles in the Streptomyces strains by $87.5 \%$ and $90.2 \%$, respectively. ${ }^{3}$ We defined this specific co-culture method as combined culture, which is conducted between actinomycetes and partner mycolic acid-containing bacteria for the efficient production of secondary metabolites. When we used a combined culture for antibiotic screening, novel bioactive compounds such as alchivemycins from $S$. endus ${ }^{5,6}$ and arcyriaflavin E from S. cinnamoneus ${ }^{7}$ were discovered.

In this study, we investigated the application range of combined culture to heterologous metabolite expression in S. lividans, a widely used host strain. We used the biosynthetic gene clusters for goadsporin, staurosporine and rebeccamycin, which we cloned previously, ${ }^{8-10}$ and demonstrated that the production of these bioactive natural products in combined cultures was increased markedly relative to those in pure cultures.

Goadsporin is a linear azole-containing peptide ${ }^{11}$ produced by Streptomyces sp. TP-A0584 and is a morphology- and secondary metabolism-inducing substance against a broad spectrum of Actinomycetales. ${ }^{12}$ (Figure 1) Goadsporin is a ribosomally synthesized and post-translationally modified peptide (RiPP), and its biosynthetic gene cluster consists of 10 genes ( $\operatorname{god} A-I)$, which include one gene for the precursor peptide $(\operatorname{god} A)$ and five genes for the post-translationally modification $($ godD $-H){ }^{8}$ The heterologous production of goadsporin in S. lividans has been previously achieved using the chromosomeintegrating cosmid vector pTOYAMAcos. ${ }^{10}$

We first conducted combined culture using the goadsporin heterologous production strain (S. lividans $\mathrm{GSBC} 1)^{8}$ with partner mycolic acid-containing bacterial strains T. pulmonis TP-B0596, R. erythropolis PR4 NBRC100887 and C. glutamicum ATCC 13869. Heterologous expression strains were first cultured in V-22 medium $^{3}$ with $20 \mu \mathrm{g} \mathrm{ml}^{-1}$ thiostrepton at $30^{\circ} \mathrm{C}$ for 3 days on a rotary shaker at

${ }^{1}$ Graduate School of Agricultural and Life Sciences, The University of Tokyo, Bunkyo, Tokyo, Japan and ${ }^{2}$ Department of Biotechnology, Toyama Prefectural University, Imizu, Toyama, Japan

Correspondence: Professor H Onaka, Graduate school of Agricultural and Life Sciences, The University of Tokyo, 1-1-1 Yayoi, Bunkyo, Tokyo 113-8657, Japan. E-mail: aonaka@mail.ecc.u-tokyo.ac.jp

Received 11 September 2014; revised 26 February 2015; accepted 26 February 2015; published online 1 April 2015 


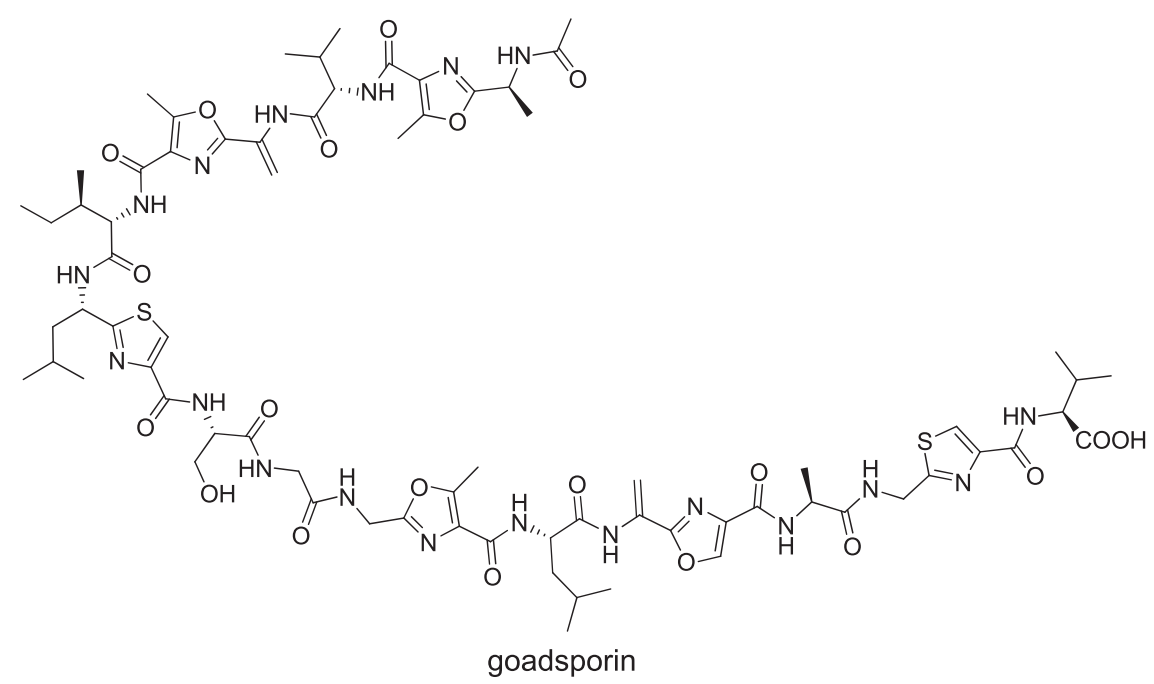<smiles></smiles>

rebeccamycin<smiles>CN[C@H]1C[C@H]2O[C@](C)([C@@H]1OC)n1c3ccccc3c3c4c(c5c6ccccc6n2c5c31)C(=O)NC4</smiles>

staurosporine

Figure 1 Chemical structures of natural products used in this study.

200 r.p.m., and the mycolic acid-containing bacteria were cultured in $\mathrm{V}-22$ medium at $30^{\circ} \mathrm{C}$ for 2 days on a rotary shaker at 200 r.p.m. for seed culture. Three milliliters of the heterologous expression strain culture and $1 \mathrm{ml}$ of the mycolic acid-containing bacterium culture were transferred to the same $500-\mathrm{ml} \mathrm{K}-1$ flask containing $100 \mathrm{ml}$ of A-3M medium ${ }^{3}$, and the two bacterial strains were grown at $30{ }^{\circ} \mathrm{C}$ on a rotary shaker at 200 r.p.m.

Goadsporin was extracted from the combined culture, and the production amounts from days 2 to 13 were measured (Figure 2a). The amounts of goadsporin in combined culture with T. pulmonis and $R$. erythropolis were higher than that of the pure culture during the entire measurement period, whereas the amount produced in combined culture with C. glutamicum was almost the same as that produced in the pure culture. The amount produced in combined culture with $T$. pulmonis reached $408 \mathrm{mgl}^{-1}$ on day 12 , and the amount of $R$. erythropolis produced in combined culture reached $233 \mathrm{mgl}^{-1}$ on day 13. Our investigation revealed that the heterologous production of goadsporin in S. lividans was improved by combined culture with $T$. pulmonis and $R$. erythropolis.

We next evaluated heterologous production of other compounds in combined culture. Staurosporine and rebeccamycin are produced by Streptomyces sp. TP-A $0274^{9}$ and Lechevalieria aerocolonigenes ATCC $39243,{ }^{13}$ respectively, and are compounds that belong to the indolocarbazole family of natural products (Figure 1). Rebeccamycin is an inhibitor of DNA topoisomerase I, whereas staurosporine is a potent protein kinase $\mathrm{C}$ inhibitor. These compounds are promising antitumor drug candidates, and several derivatives are currently in clinical trials. $^{14,15}$ The staurosporine and rebeccamycin biosynthetic gene clusters consist of 15 and 11 genes, respectively. We constructed heterologous production mutants for both compounds as described in our previous study. ${ }^{9,13}$ The cosmid clones pTOYAMA-Sta containing the staurosporine biosynthetic gene cluster and pTOYAMA-Reb containing the rebeccamycin biosynthetic gene cluster were transconjugated into $S$. lividans TK23, and these transformants were combined cultured. We compared the amounts of staurosporine and rebeccamycin produced in combined culture and pure culture. The production of these two indolocarbazoles in pure culture gave fairly low yields, whereas the production of each antibiotic in combined culture was significantly higher in comparison (Figures $2 b$ and c).

The amount of staurosporine produced in the pure culture reached only $0.6 \mathrm{mgl}^{-1}$, and the yields did not increase during the entire measurement period; however, the concentration in the combined culture started to increase after day 2 (Figure $2 \mathrm{~b}$ ). The maximum yield of staurosporine in the combined culture with $T$. pulmonis was reached on day 12, which produced $104 \mathrm{mgl}^{-1}$, 200-fold higher than the amount produced in the pure culture. On day 12, combined culture with $R$. erythropolis produced 39-fold more staurosporine than the pure culture and that with C. glutamicum produced 12-fold more than the pure culture.

The amount of rebeccamycin produced in the pure culture remained relatively low until day 7 , whereas combined culture with the three mycolic acid-containing bacteria produced $>1.6 \mathrm{mgl}^{-1}$ (Figure 2c). On day 7, the combined culture amounts produced were much higher than that of the pure culture, which produced yields of 130-fold with T. pulmonis, 60-fold with R. erythropolis and 16-fold with C. glutamicum. After day 8, the pure culture started producing 

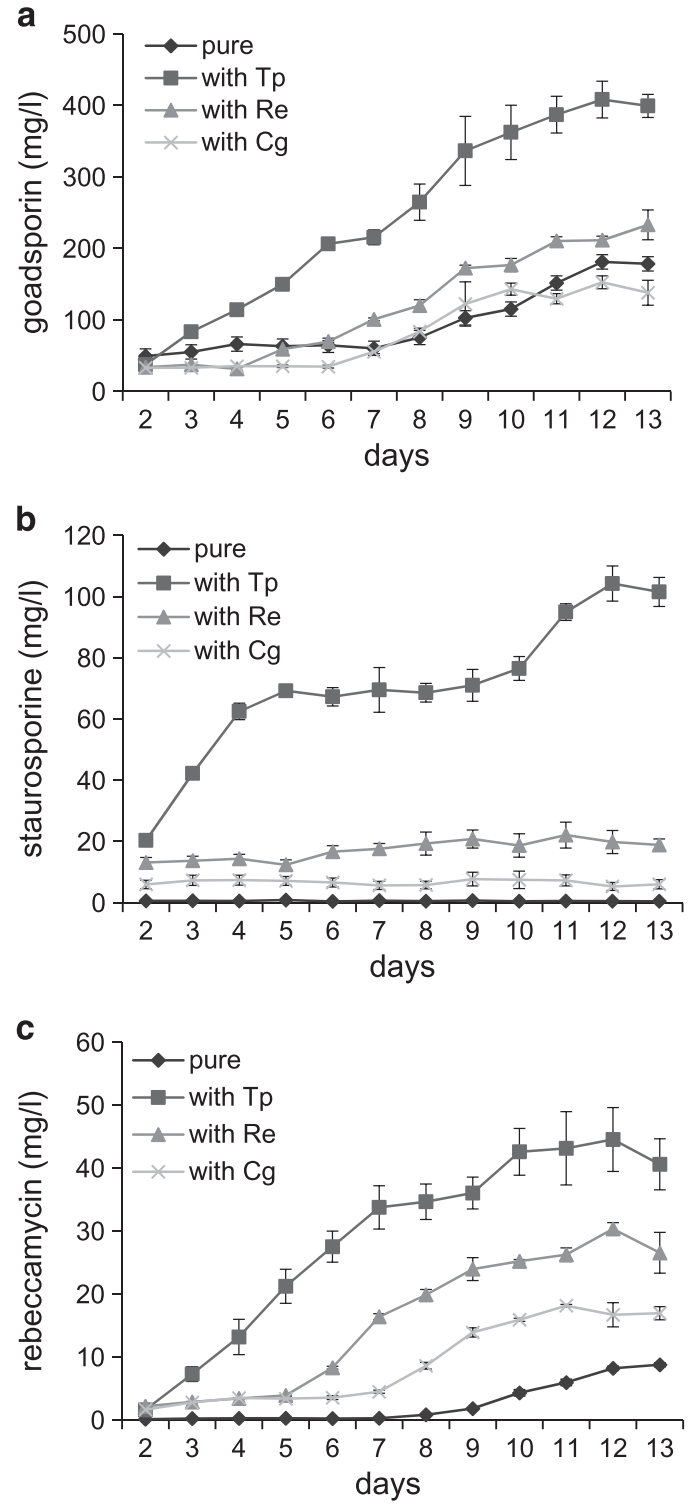

Figure 2 Production traces of (a) goadsporin, (b) staurosporine and (c) rebeccamycin in combined culture with $T$. pulmonis $(\mathrm{Tp}), R$. erythropolis $(\mathrm{Re})$ and $C$. glutamicum $(\mathrm{Cg})$ compared with those in pure culture. The culture broth was extracted with an equal volume of $n$-butanol. The $n$-butanol extract was then injected into an HPLC system (HP1200; Hewlett Packard, CA, USA). The sample was separated on a COSMOSIL $5 \mathrm{C}_{18}$ AR-II column $(5 \mu \mathrm{m}$, $2.0 \mathrm{~mm}$ i.d. $\times 150 \mathrm{~mm}$, Nacalai Tesque, Kyoto, Japan). Acetonitrile and $0.1 \%$ formic acid were used as the elution solvents. The column temperature was kept at $40^{\circ} \mathrm{C}$, and the flow rate was $0.3 \mathrm{ml} \mathrm{min}^{-1}$. The concentration of acetonitrile was kept at $5 \%$ for the first 2 min, linearly increased to $95 \%$ over the next $25 \mathrm{~min}$, and kept at $95 \%$ for the next $5 \mathrm{~min}$. Metabolite profiles were monitored at $254 \mathrm{~nm}$ (for goadsporin), $292 \mathrm{~nm}$ (for staurosporine) and $314 \mathrm{~nm}$ (for rebeccamycin). The amounts of goadsporin, staurosporine and rebeccamycin were quantified using authentic standards. Staurosporine and rebeccamycin were purchased from Sigma-Aldrich (Tokyo, Japan). Chart legends: with $\mathrm{Tp}$, with $\mathrm{Re}$ and with $\mathrm{Cg}$ represent combined culture with T. pulmonis, $R$. erythropolis and C. glutamicum, respectively. A full color version of this figure is available at The Journal of Antibiotics journal online.

rebeccamycin but the levels paled in comparison with those of the combined cultures. The maximum production of rebeccamycin was achieved in combined culture with $T$. pulmonis, reaching $44.5 \mathrm{mg} \mathrm{l}^{-1}$ on day 12.
The heterologous expression system is a well-established technique for producing secondary metabolites. However, the production levels are frequently insufficient for detecting the heterologously produced compounds. In this study, we evaluated the yields of two types of natural products, RiPPs ${ }^{11}$ and indolocarbazoles, in combined culture. Our results clearly showed that the yields of both product types could be increased by combined culture. In addition, the production of these products in combined culture was initiated earlier than in pure culture. Therefore, combined culture is an effective and applicable method for increasing the yield of heterologous expression products in S. lividans.

Combined culture affects a variety of secondary metabolisms not only in S. lividans but also in a variety of Streptomyces species. ${ }^{3}$ It was rather surprising that products biosynthesized by heterologous gene clusters, which are also regulated by different types of Streptomyces antibiotic regulatory proteins, are increased in combined culture. As the production of pigments was also observed in heterologous production hosts (data not shown) during culturing, which is triggered by the physical contact of the two bacteria, the same mechanism may be involved in incrementing heterologous metabolite production. One possibility is that mycolic acid-containing bacteria stimulate the upper regulatory system, which may be globally conserved in Streptomyces strains. In addition to activation mediated by cell-to-cell interactions, mycolic acid-containing bacteria may have a role as a supplier of digested nutrients or substrates for $S$. lividans for the production of heterologous antibiotics. Further transcriptional, translational and physiological analyses would be required for characterization of these bacterial stimuli.

As combined culture positively regulates the production of the three compounds examined in this study, this method would also be applicable for secondary metabolites encoded in silent gene clusters. Combined culture, which requires only the addition of mycolic acidcontaining bacteria to the pure culture, can activate secondary metabolism without complicated genetic manipulation or the addition of chemical inducers for secondary metabolism. Thus, this method may be advantageous for scaling up fermentation volumes and is an efficient strategy for the identification and production of various secondary metabolites.

\section{ACKNOWLEDGEMENTS}

This work was supported in part by a Grant-in-Aid from the Institute for Fermentation, Osaka (HO, TO and SA), and by JSPS KAKENHI Grants $25108707(\mathrm{HO})$ and $90709057(\mathrm{SA})$.

1 Ikeda, H., Shin-ya, K. \& Omura, S. Genome mining of the Streptomyces avermitilis genome and development of genome-minimized hosts for heterologous expression of biosynthetic gene clusters. J. Ind. Microbiol. Biotechnol. 41, 233-250 (2014).

2 Bertrand, S., Bohni, N., Schnee, S., Schumpp, O., Gindro, K. \& Wolfender, J. L. Metabolite induction via microorganism co-culture: a potential way to enhance chemical diversity for drug discovery. Biotechnol. Adv. 32, 1180-1204 (2014).

3 Onaka, H., Mori, Y., Igarashi, Y. \& Furumai, T. Mycolic acid-containing bacteria induce natural-product biosynthesis in Streptomyces species. Appl. Environ. Microbiol. 77 400-406 (2011).

4 Marrakchi, H., Laneelle, M. A. \& Daffe, M. Mycolic acids: structures, biosynthesis, and beyond. Chem. Biol. 21, 67-85 (2014).

5 Igarashi, Y. et al. Alchivemycin A, a bioactive polycyclic polyketide with an unprecedented skeleton from Streptomyces sp. Org. Lett. 12, 3402-3405 (2010).

$6 \mathrm{Kim}$, Y., In, Y., Ishida, T., Onaka, H. \& Igarashi, Y. Biosynthetic origin of alchivemycin A, a new polyketide from Streptomyces and absolute configuration of alchivemycin B. Org. Lett. 15, 3514-3517 (2013).

7 Hoshino, S., Zhang, L., Awakawa, T., Wakimoto, T., Onaka, H. \& Abe, I. Arcyriaflavin E, a new cytotoxic indolocarbazole alkaloid isolated by combined-culture of mycolic acidcontaining bacteria and Streptomyces cinnamoneus NBRC 13823. J. Antibiot. 68, 342-344 (2015). 
8 Onaka, H., Nakaho, M., Hayashi, K., Igarashi, Y. \& Furumai, T. Cloning and characterization of the goadsporin biosynthetic gene cluster from Streptomyces sp. TP-A0584. Microbiology 151, 3923-3933 (2005).

9 Onaka, H., Taniguchi, S., Igarashi, Y. \& Furumai, T. Cloning of the staurosporine biosynthetic gene cluster from Streptomyces sp. TP-A0274 and its heterologous expression in Streptomyces lividans. J. Antibiot. (Tokyo) 55, 1063-1071 (2002).

10 Onaka, H., Taniguchi, S., Ikeda, H., Igarashi, Y. \& Furumai, T. pTOYAMAcos, pTYM18, and PTYM19, actinomycete-Escherichia coli integrating vectors for heterologous gene expression. J. Antibiot. (Tokyo) 56, 950-956 (2003).

11 Arnison, P. G. et al. Ribosomally synthesized and post-translationally modified peptide natural products: overview and recommendations for a universal nomenclature. Nat. Prod. Rep. 30, 108-160 (2013)
12 Onaka, H., Tabata, H., Igarashi, Y., Sato, Y. \& Furumai, T. Goadsporin, a chemical substance which promotes secondary metabolism and morphogenesis in streptomycetes. I. Purification and characterization. J. Antibiot. (Tokyo) 54 1036-1044 (2001).

13 Onaka, H., Taniguchi, S., Igarashi, Y. \& Furumai, T. Characterization of the biosynthetic gene cluster of rebeccamycin from Lechevalieria aerocolonigenes ATCC 39243. Biosci. Biotechnol. Biochem. 67, 127-138 (2003).

14 Sanchez, C., Mendez, C. \& Salas, J. A. Indolocarbazole natural products: occurrence, biosynthesis, and biological activity. Nat. Prod. Rep. 23, 1007-1045 (2006).

15 Nakano, H. \& Omura, S. Chemical biology of natural indolocarbazole products: 30 years since the discovery of staurosporine. J. Antibiot. (Tokyo) 62, 17-26 (2009). 